\title{
Local Magnetic Order vs Superconductivity in a Layered Cuprate
}

\author{
N. Ichikawa, ${ }^{1} *$ S. Uchida, ${ }^{1}$ J. M. Tranquada, ${ }^{2}$ T. Niemöller, ${ }^{3}$ P. M. Gehring, ${ }^{4}$ S.-H. Lee, ${ }^{4,5}$ and J. R. Schneider ${ }^{3}$ \\ ${ }^{1}$ Department of Superconductivity, School of Engineering, University of Tokyo, 2-11-16 Yayoi, Bunkyo-ku, Tokyo 113-8656, Japan \\ ${ }^{2}$ Physics Department, Brookhaven National Laboratory, Upton, New York 11973-5000 \\ ${ }^{3}$ Hamburger Synchrotronstrahlungslabor HASYLAB at Deutsches Elektronen-Synchrotron DESY, \\ Notkestrasse 85, D-22603 Hamburg, Germany \\ ${ }^{4}$ NIST Center for Neutron Research, National Institute of Standards and Technology, Gaithersburg, Maryland 20899 \\ ${ }^{5}$ University of Maryland, College Park, Maryland 20742
}

(Received 4 October 1999; revised manuscript received 9 February 2000)

\begin{abstract}
We report on the phase diagram for charge-stripe order in $\mathrm{La}_{1.6-x} \mathrm{Nd}_{0.4} \mathrm{Sr}_{x} \mathrm{CuO}_{4}$, determined by neutron and $\mathrm{x}$-ray scattering studies and resistivity measurements. From an analysis of the in-plane resistivity motivated by recent nuclear-quadrupole-resonance studies, we conclude that the transition temperature for local charge ordering decreases monotonically with $x$, and hence that local antiferromagnetic order is uniquely correlated with the anomalous depression of superconductivity at $x \approx \frac{1}{8}$. This result is consistent with theories in which superconductivity depends on the existence of charge-stripe correlations.
\end{abstract}

PACS numbers: 74.25.Fy, 71.45.Lr, 74.72.Dn, 75.30.Fv

Superconductivity in the layered cuprates is induced by doping charge carriers into an antiferromagnetic insulator. The kinetic energy of the mobile carriers competes with the superexchange interaction between neighboring $\mathrm{Cu}$ spins $[1,2]$. There is increasing evidence for the hole-doped cuprates that this competition drives a spatial segregation of holes which form antiphase domain walls between strips of antiferromagnetically correlated $\mathrm{Cu}$ spins [3-8]. A major controversy surrounds the issue of whether the mesoscopic self-organization of charges and spins is a necessary precursor for hightemperature superconductivity [9-11] or whether it is simply an alternative instability that competes with superconductivity $[12,13]$.

To gain further insight into this problem, we have performed a systematic study of the phase diagram of $\mathrm{La}_{1.6-x} \mathrm{Nd}_{0.4} \mathrm{Sr}_{x} \mathrm{CuO}_{4}$ (LNSCO), a system for which evidence of competition between superconductivity and stripe order has been reported previously [14]. From neutron and x-ray scattering measurements we show that the charge and magnetic ordering temperatures reach their maxima at $x \approx \frac{1}{8}$. For $x<\frac{1}{8}$, the charge-ordering transition is limited by a structural phase boundary. The low-temperature structural phase involves a change in the tilt pattern of the $\mathrm{CuO}_{6}$ octahedra, stabilized by the substituted $\mathrm{Nd}$, which can pin vertical charge stripes [15].

At first glance, these results, together with the anomalous depression of the superconducting transition temperature, $T_{c}$, at $x \approx \frac{1}{8}$, appear to provide confirmation that charge-stripe order is in direct competition with superconductivity; however, the picture becomes more complicated when one takes into account recent nuclear-quadrupoleresonance (NQR) studies of LNSCO [4,5]. In this work, a transition (involving the onset of an apparent loss of intensity) has been identified which coincides with the charge ordering determined by diffraction for $x \geq \frac{1}{8}$; however, in contrast to the diffraction results, the NQR transition temperature, $T_{\mathrm{NQR}}$, continues to increase as $x$ decreases below $\frac{1}{8}$. Furthermore, the same transition is observed in $\mathrm{La}_{2-x} \mathrm{Sr}_{x} \mathrm{CuO}_{4}$ (LSCO) for $x \lessgtr \frac{1}{8}$. The implication is that local charge order, not easily detected by diffraction techniques, may occur even in the absence of the Nd-stabilized lattice modulation.

Does $T_{\mathrm{NQR}}$ really correspond to charge ordering? To test this possibility, we have analyzed the in-plane resistivity, $\rho_{a b}(T)$, which should be a sensitive measure of charge ordering. Through a scaling analysis, we have identified a temperature scale $T_{u}$ that corresponds to a low-temperature upturn with respect to an extrapolated linear variation with $T$. (The signature of the charge ordering is subtle, as befitting its unconventional nature.) We show that $T_{u}$ corresponds with $T_{\mathrm{NQR}}$ for both LNSCO and LSCO, thus providing support for the association of $T_{\mathrm{NQR}}$ with local charge ordering. Furthermore, we show that $T_{u}$ and $T_{\mathrm{NQR}}$ are linearly correlated with the size of the lattice distortion at low temperature. Together with the reasonable assumption that the magnitude of the charge-order parameter at low temperature is correlated with the ordering temperature, this result is strong evidence for a monotonic decrease of the charge-order parameter with increasing hole concentration (over the range studied here).

A monotonic variation of the stripe pinning strength means that there is no correlation with the anomalous depression of $T_{c}$ at $x \approx \frac{1}{8}$. We are left with the surprising conclusion that it is, instead, the static magnetic order alone which has a special association with the $\frac{1}{8}$ anomaly. In making this assertion, we do not argue that ordering the charge is good for superconductivity; to the contrary, $T_{c}$ is certainly reduced in all of our LNSCO samples compared to comparably doped LSCO. Rather, our point is that, while pinning charge stripes is not good, it is magnetic order that is truly incompatible with superconductivity. The competition between static local antiferromagnetism and superconductivity is supported by recent theoretical 
work [16] and is compatible with the spin-gap proximityeffect mechanism for superconductivity [9].

For this study, a series of crystals of $\mathrm{La}_{2-x-y} \mathrm{Nd}_{y}-$ $\mathrm{Sr}_{x} \mathrm{CuO}_{4}$, with $y=0.4$ and $x=0.08$ to 0.25 , was grown by the traveling-solvent floating-zone method [17]. Figure 1(a) shows the electrical resistivity measured parallel to the $\mathrm{CuO}_{2}$ planes by the six-probe method. As previously reported [18], there are upturns in $\rho_{a b}$ at low temperature for the $x=0.12$ and 0.15 samples, compositions at which charge order has been observed $[3,15,18,19]$. In each there is also a small jump near $70 \mathrm{~K}$, where a subtle structural transition takes place from the socalled low-temperature-orthorhombic (LTO) phase to the low-temperature-tetragonal (LTT) or an intervening lowtemperature-less-orthorhombic (LTLO) phase [20,21]. At $x=0.12$, charge ordering and the structural transition are essentially coincident $[18,19]$; however, charge ordering occurs significantly below the structural phase change at $x=0.15$ (see Fig. 2) [3].

The resistivity for $x=0.10$ looks somewhat different. Instead of an increase at the structural transition temperature, $\rho_{a b}$ decreases below the transition and continues to
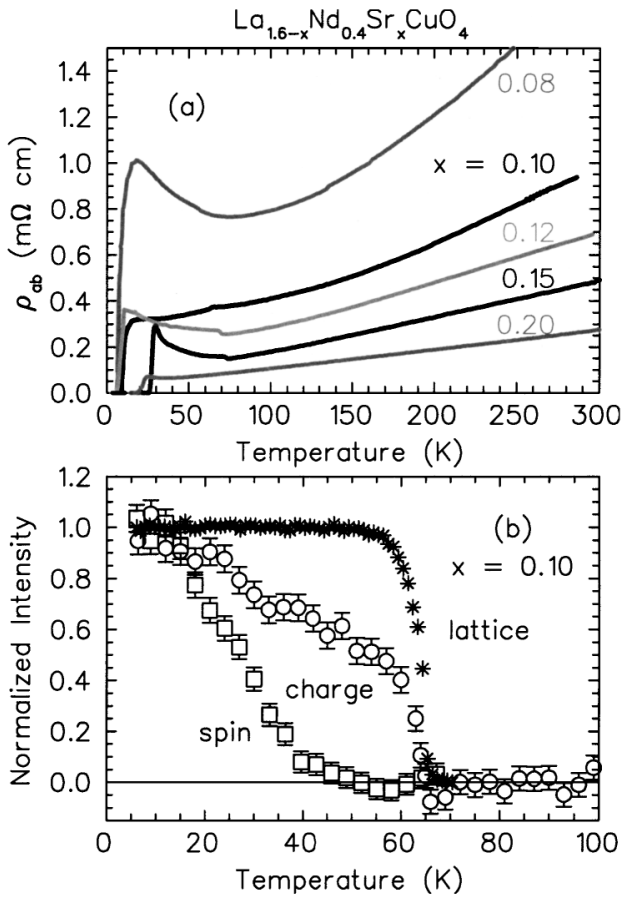

FIG. 1. (a) In-plane resistivity vs temperature measured on single crystals of $\mathrm{La}_{1.6-x} \mathrm{Nd}_{0.4} \mathrm{Sr}_{x} \mathrm{CuO}_{4}$ with several different $\mathrm{Sr}$ concentrations. (b) Neutron diffraction results for $x=0.10$. Stars: intensity of the (100) superlattice peak, which is allowed in the LTT and LTLO phases, but not in LTO. Circles: Chargeorder superlattice peak at wave vector $(2+2 \epsilon, 0,0.5)$. Squares: Magnetic superlattice peak at $\left(\frac{1}{2}+\epsilon, \frac{1}{2}, 0\right)$. (Peak indexing is based on the simple tetragonal unit cell [15].) In each case, peak intensity was measured by scanning $T$ without moving the spectrometer. $T$ dependence of the background was measured and subtracted, and a $T$-independent offset was applied. decrease in a typically metallic fashion until superconductivity sets in. To test whether stripe order occurs in this sample, we performed a neutron scattering experiment at the NIST Center for Neutron Research (NCNR) [23]. We found that the $x=0.10$ sample does indeed exhibit charge and spin order. The temperature dependence of the peak intensities for representative superlattice peaks are shown in Fig. 1(b). On warming, the charge order (which has also been confirmed by $x$-ray diffraction measurements at HASYLAB) seems to be limited by the structural transition at $65 \mathrm{~K}$, while the magnetic order disappears at a lower temperature.

We have also used neutron scattering to determine the magnetic ordering temperatures $\left(T_{m}\right)$ in samples with $x=$ 0.08 and 0.25. The results are summarized in Fig. 2 . (Further details of the neutron studies will be presented elsewhere.) The new results for $x=0.08$ and 0.10 make it clear that the highest $T_{m}$ occurs at $x \sim \frac{1}{8}$, where the superconducting transition $\left(T_{c}\right)$ is most greatly depressed. Also plotted in the figure are the transition temperatures $\left(T_{\mathrm{NQR}}\right)$ deduced from $\mathrm{Cu} \mathrm{NQR}$ measurements by Singer and co-workers $[4,5]$. Those temperatures coincide with the charge-order transitions, $T_{\mathrm{ch}}$, for $x=0.12$ and 0.15

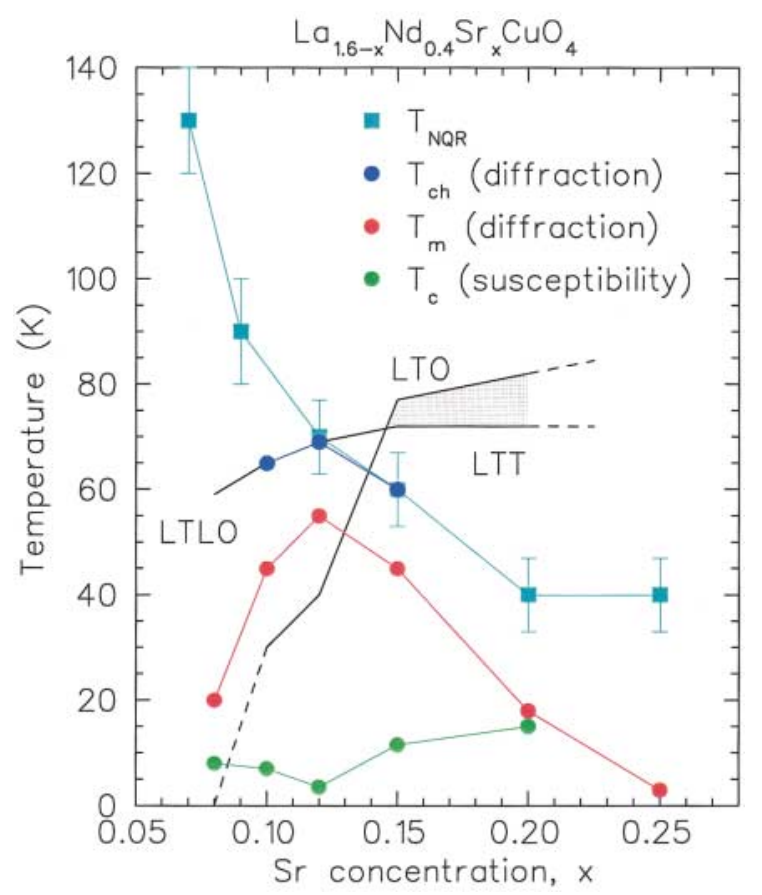

FIG. 2 (color). Phase diagram for $\mathrm{La}_{1.6-x} \mathrm{Nd}_{0.4} \mathrm{Sr}_{x} \mathrm{CuO}_{4}$. Light-blue squares: $T_{\mathrm{NQR}}$ [5]; dark-blue circles: $T_{\mathrm{ch}}$ from diffraction studies $[3,18,19]$ (and present work); red circles: $T_{m}$ from neutron diffraction [14,17]; green circles: $T_{c}$ from magnetic susceptibility [14,17,22]. Lines through symbols are guides to the eye. Black lines indicate structural phase boundaries determined by neutron diffraction [17,18]. Shaded region indicates coexistence of LTO and LTT phases. The $x=0.25$ crystal appears to be a mixture of LTO and LTT phases with no obvious transition between 10 and $300 \mathrm{~K}$. 
determined by diffraction, but there appears to be a discrepancy for $x<0.12$.

The NQR and diffraction results for $x<0.12$ are not necessarily in conflict, since NQR is an inherently local probe, whereas the diffraction measurements require substantial spatial correlations of the charge order in order to obtain detectable superstructure peaks. But it is also interesting that NQR measurements $[4,5]$ suggest charge order in pure $\mathrm{La}_{2-x} \mathrm{Sr}_{x} \mathrm{CuO}_{4}$ for $x \lessgtr 0.125$, where diffraction studies have not yet detected any charge-related superlattice peaks. If some form of charge ordering is occurring within the LTO phase, one would expect to see an indication of it in the resistivity. As we show below, it is, in fact, possible to identify a signature of charge order in resistivity measurements.

To analyze the resistivity, we consider first the behavior at higher temperatures. For cuprates doped to give the maximum $T_{c}$, it was noted early on [24] that, over a surprisingly large temperature range,

$$
\rho(T)=\alpha T+\beta,
$$

with $\beta$ very close to zero. We find that this formula describes fairly well the results in Fig. 1(a) for $T \gtrsim 200 \mathrm{~K}$. Values for $\alpha$ were obtained by fitting Eq. (1), with $\beta \equiv 0$, to data in the range $250<T<300 \mathrm{~K}$; the same analysis was also applied to resistivity data for $\mathrm{La}_{2-x} \mathrm{Sr}_{x} \mathrm{CuO}_{4}$ crystals with $x=0.10,0.12,0.15$, and 0.20 [17].

Next, we analyze the upturn in the resistivity at low temperature. The temperature at which the upturn becomes significant varies with $x$ and so does the rate of upturn; it was pointed out previously by Büchner and co-workers [25] that the rate of upturn increases monotonically as one goes from $x=0.10$ to 0.12 to 0.15 . We have found that all of the data can be scaled approximately onto a single curve if $\rho_{a b}$ is divided by $\alpha T$ and then plotted against a reduced temperature $t=\left(T-T_{0}\right) / T_{u}$, where $T_{u}$ is the characteristic upturn temperature and $T_{0}$ is the temperature towards which $\rho_{a b}$ appears to be diverging. The scaled resistivities are shown in Fig. 3; note that the same scaling is useful for samples both with and without Nd. The scaled curve is given approximately by

$$
\rho_{a b} / \alpha T=\tanh (15 t) / \tanh (t),
$$

and we have determined error bars for the parameters $T_{0}$ and $T_{u}$ by performing least-squares fits to this function.

The values of $T_{u}$ are compared with $T_{\mathrm{NQR}}$ in Fig. 4, where both are plotted vs the maximum orthorhombic splitting $(b-a)_{\text {LTO }}$ in the LTO phase. Büchner et al. [26] have shown that $(b-a)_{\text {LTO }}$ is a useful measure of the octahedral tilt angle, which changes orientation but not magnitude in the LTLO and LTT phases. For the $y=$ 0.4 samples, we used our own neutron measurements of $(b-a)_{\text {LTO }}$, while we used results from [27] for LSCO.

From Fig. 4 we see that (i) the values of $T_{u}$ and $T_{\mathrm{NQR}}$ agree within the error bars and (ii) both values tend to scale with the octahedral tilt angle, independent of the tilt

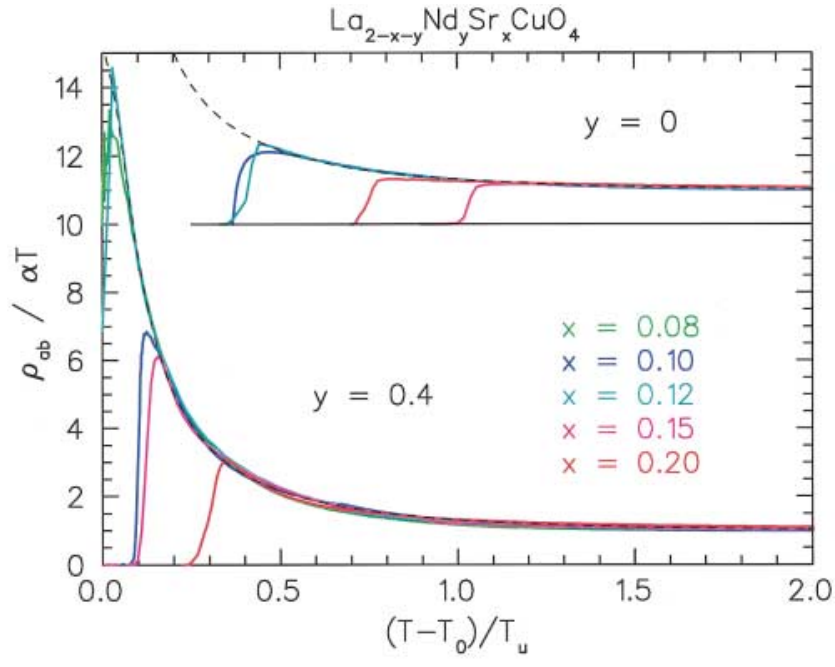

FIG. 3 (color). $\quad \rho_{a b}$ divided by $\alpha T$ vs $\left(T-T_{0}\right) / T_{u}$ for $\mathrm{La}_{2-x-y} \mathrm{Nd}_{y} \mathrm{Sr}_{x} \mathrm{CuO}_{4}$ with $y=0.4$ and $y=0$ (shifted vertically). The black dashed line is a model function described in the text.

orientation (LTO vs LTT). The first point reinforces the association of $T_{\mathrm{NQR}}$ with charge order, while the second indicates that the ordering temperature for local charge ordering is controlled by the tilt angle. (A correlation between tilt angle and $T_{c}$ reduction was noted previously by Dabrowski et al. [28].) Longer-range charge correlations (those detected by diffraction) appear to be sensitive to the tilt orientation.

The variation of $T_{0}$ with $x$ is shown in the inset in Fig. 4 for $y=0.4$. There is a considerable increase in $T_{0}$ from $x=0.10$ to 0.15 . We suggest that this trend may be associated with a phase locking of charge-density-wave correlations along neighboring charge stripes, a possibility suggested by Kivelson, Fradkin, and Emery [29]. Whether or not this interpretation is correct, there is clearly no correlation between the variations of $T_{0}$ (or $T_{u}$ ) and the depression of $T_{c}$ for $y=0.4$, which is greatest at $x \approx \frac{1}{8}$.

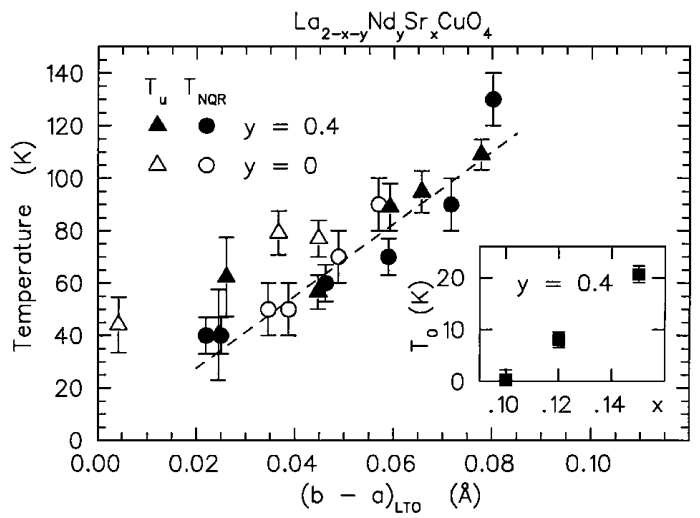

FIG. 4. Values of parameters $T_{u}$ (triangles) and $T_{\mathrm{NQR}}$ (circles) as a function of $(b-a)_{\mathrm{LTO}}$ for $\mathrm{La}_{2-x-y} \mathrm{Nd}_{y} \mathrm{Sr}_{x} \mathrm{CuO}_{4}$ (filled symbols, $y=0.4$; open, $y=0$ ). Dashed line is a guide to the eye. Inset: $T_{0}$ vs $x$ for $y=0.4$. 
The variations of $T_{u}$ and $T_{0}$ shown in Fig. 4 strongly indicate that ordering of the charge stripes is not responsible for the strong depression of $T_{c}$ at $x \approx \frac{1}{8}$. We are then left with the conclusion that the culprit must be the magnetic order, which is maximized at the point where $T_{c}$ is minimized. That local antiferromagnetic order competes with superconductivity is certainly compatible with the spin-gap proximity-effect mechanism for superconductivity [9]. In that theory, hole pairing is associated with the occurrence of a spin gap; given that antiferromagnetic order competes with singlet correlations and a spin gap, one would then expect $T_{c}$ to be depressed when magnetic order is present. (Of course, charge order is a prerequisite for magnetic order.) The trade-off between local magnetic order and superconductivity is also emphasized in a recent numerical study [16].

One simple reason why $T_{m}$ might reach a maximum at $x=\frac{1}{8}$ is suggested by recent analyses of coupled spin ladders [30,31]. If the charge stripes are rather narrow and centered on rows of $\mathrm{Cu}$ atoms, then the intervening magnetic strips would consist of three-leg spin ladders. Theoretical analyses have shown that even weak couplings between a series of three-leg ladders will lead to order at sufficiently low temperature, whereas weakly coupled twoor four-leg ladders have a quantum-disordered ground state [30,31]. As $x$ deviates from $\frac{1}{8}$, one would have a combination of even-leg and three-leg ladders, thus weakening the tendency to order. Although there is no direct experimental evidence concerning the registry of the stripes with the lattice, the picture of $\mathrm{CuO}_{2}$ plane broken into a series of three-leg ladders by $\mathrm{Cu}$-centered charge stripes at $x=\frac{1}{8}$ is appealing in the present case.

One might argue that only longer-range magnetic (or charge) order is relevant for suppressing superconductivity. We believe that a counterexample is given by the case of $\mathrm{Zn}$ doping, where a local suppression of superconductivity is associated with static short-range antiferromagnetic correlations about the $\mathrm{Zn}$ sites [32].

In conclusion, we have presented evidence that it is local magnetic order rather than charge-stripe order which is responsible for the anomalous suppression of superconductivity in LNSCO at $x \approx \frac{1}{8}$. While pinning charge stripes also causes some reduction of $T_{c}$, charge order appears to be compatible with superconductivity as long as the spin correlations remain purely dynamic.

This research was supported by the U.S.-Japan Cooperative Research Program on Neutron Scattering, by a COE Grant from the Ministry of Education, Japan, and by U.S. Department of Energy Contract No. DE-AC0298CH10886. We acknowledge the support of the NIST, U.S. Department of Commerce, in providing the neutron facilities used in this work; SPINS is supported by the National Science Foundation under Agreement No. DMR-
9423101. N. I. and J. M. T. acknowledge the hospitality of the NCNR staff. We thank V. J. Emery and S. A. Kivelson for helpful comments.

*Present address: Institute for Chemical Research, Kyoto University, Uji 611-0011, Japan; CREST, Japan Science and Technology Corporation (JST), Kawaguchi, Saitama 332-0012, Japan.

[1] P. W. Anderson, Adv. Phys. 46, 3 (1997).

[2] V. J. Emery, S. A. Kivelson, and J. M. Tranquada, Proc. Natl. Acad. Sci. U.S.A. 96, 8814 (1999).

[3] T. Niemöller et al., Eur. Phys. J. B 12, 509 (1999).

[4] A. W. Hunt, P. M. Singer, K. R. Thurber, and T. Imai, Phys. Rev. Lett. 82, 4300 (1999).

[5] P. M. Singer, A. W. Hunt, A. F. Cederström, and T. Imai, Phys. Rev. B 60, 15345 (1999).

[6] S. Wakimoto et al., Phys. Rev. B 60, R769 (1999).

[7] H. A. Mook et al., Nature (London) 395, 580 (1998).

[8] H. A. Mook and F. Doğan, Nature (London) 401, 145 (1999).

[9] V. J. Emery, S. A. Kivelson, and O. Zachar, Phys. Rev. B 56, 6120 (1997).

[10] C. Castellani, C. Di Castro, and M. Grilli, Z. Phys. B 103, 137 (1997).

[11] M. Vojta and S. Sachdev, Phys. Rev. Lett. 83, 3916 (1999).

[12] T. M. Rice, Physica (Amsterdam) 282C-287C, xix (1997).

[13] G. Baskaran, cond-mat/9910161.

[14] J. M. Tranquada et al., Phys. Rev. Lett. 78, 338 (1997).

[15] J. M. Tranquada et al., Nature (London) 375, 561 (1995).

[16] M. Havilio and A. Auerbach, Phys. Rev. Lett. 83, 4848 (1999).

[17] N. Ichikawa, Ph.D. thesis, University of Tokyo, 1999.

[18] J. M. Tranquada et al., Phys. Rev. B 54, 7489 (1996).

[19] M.v. Zimmermann et al., Europhys. Lett. 41, 629 (1998).

[20] J. D. Axe et al., Phys. Rev. Lett. 62, 2751 (1989).

[21] M. K. Crawford et al., Phys. Rev. B 44, 7749 (1991).

[22] J.E. Ostenson et al., Phys. Rev. B 56, 2820 (1997).

[23] The $x=0.10$ sample was studied at spectrometer BT4 with $14.7-\mathrm{meV}$ neutrons; $x=0.08$ and 0.25 samples were characterized at the NG-5 SPINS spectrometer with 5-meV neutrons.

[24] M. Gurvitch and A. T. Fiory, Phys. Rev. Lett. 59, 1337 (1987).

[25] M. Hücker et al., J. Phys. Chem. Solids 59, 1821 (1998).

[26] B. Büchner, M. Breuer, A. Freimuth, and A.P. Kampf, Phys. Rev. Lett. 73, 1841 (1994).

[27] P. G. Radaelli et al., Phys. Rev. B 49, 4163 (1994).

[28] B. Dabrowski et al., Phys. Rev. Lett. 76, 1348 (1996).

[29] S. A. Kivelson, E. Fradkin, and V. J. Emery, Nature (London) 393, 550 (1998).

[30] J. Tworzydło, O. Y. Osman, C. N. A. van Duin, and J. Zaanen, Phys. Rev. B 59, 115 (1999).

[31] Y. J. Kim et al., Phys. Rev. B 60, 3294 (1999).

[32] M.-H. Julien et al., Phys. Rev. Lett. 84, 3422 (2000); B. Nachumi et al., ibid. 77, 5421 (1996); T. Nakano et al., Phys. Rev. B 58, 5831 (1998). 